Review Article

\title{
Association of Cancer and the Risk of Developing Atrial Fibrillation: A Systematic Review and Meta-Analysis
}

\author{
Ming Yuan, ${ }^{1}$ Zhiwei Zhang, ${ }^{1}$ Gary Tse $\mathbb{D D}^{2,3}$ Xiaojin Feng, ${ }^{1}$ Panagiotis Korantzopoulos $\mathbb{D}^{4}{ }^{4}$ \\ Konstantinos P. Letsas, ${ }^{5}$ Bryan P. Yan, ${ }^{2,6}$ William K. K. Wu, ${ }^{7}$ Huilai Zhang, \\ Guangping Li $\mathbb{D},{ }^{1}$ Tong Liu $\mathbb{D},{ }^{1}$ and Yunlong Xia $\mathbb{D}^{9}$ \\ ${ }^{1}$ Tianjin Key Laboratory of Ionic-Molecular Function of Cardiovascular Disease, Department of Cardiology, \\ Tianjin Institute of Cardiology, Second Hospital of Tianjin Medical University, Tianjin 300211, China \\ ${ }^{2}$ Department of Medicine and Therapeutics, Chinese University of Hong Kong, Hong Kong, SAR, China \\ ${ }^{3}$ Li Ka Shing Institute of Health Sciences, 30-32 Ngan Shing St, Chinese University of Hong Kong, Hong Kong, SAR, China \\ ${ }^{4}$ First Department of Cardiology, University Hospital of Ioannina, Ioannina, Greece \\ ${ }^{5}$ Second Department of Cardiology, Laboratory of Cardiac Electrophysiology, Evangelismos General Hospital of Athens, \\ Athens, Greece \\ ${ }^{6}$ Department of Epidemiology and Preventive Medicine, Monash University, Clayton, Australia \\ ${ }^{7}$ Department of Anaesthesia and Intensive Care, Faculty of Medicine, The Chinese University of Hong Kong, Hong Kong, China \\ ${ }^{8}$ Department of Lymphoma, Tianjin Medical University Cancer Institute and Hospital, \\ National Clinical Research Center of Cancer, Key Laboratory of Cancer Prevention and Therapy, Tianjin, China \\ ${ }^{9}$ Department of Cardiology, First Affiliated Hospital of Dalian Medical University, Dalian, China
}

Correspondence should be addressed to Tong Liu; liutongdoc@126.com and Yunlong Xia; yunlong_xia@126.com

Received 5 August 2018; Revised 18 February 2019; Accepted 3 March 2019; Published 14 April 2019

Academic Editor: Robert Chen

Copyright (c) 2019 Ming Yuan et al. This is an open access article distributed under the Creative Commons Attribution License, which permits unrestricted use, distribution, and reproduction in any medium, provided the original work is properly cited.

Aims. Previous studies have demonstrated epidemiological evidence for an association between cancer and the development of new-onset atrial fibrillation (AF). However, these results have been conflicting. This systematic review and meta-analysis was conducted to examine the relationship between cancer and the risk of developing atrial fibrillation. Methods. PubMed and Web of Science were searched for publications examining the association between cancer and atrial fibrillation risk published until June 2017. Adjusted odds ratios (ORs) or hazard ratios (HRs) and 95\% CI were extracted and pooled. Results. A total of five studies involving 5,889,234 subjects were included in this meta-analysis. Solid cancer patients are at higher risk developing atrial fibrillation compared to noncancer patients (OR 1.47, 95\% CI 1.31 to $1.66, p<0.00001 ; I^{2}=67 \%, p=0.02$ ). The risk of atrial fibrillation was highest within 90 days of cancer diagnosis (OR 7.62, 95\% CI 3.08 to $18.88, p<0.00001$ ) and this risk diminished with time. Conclusions. The risk of AF was highest within 90 days of cancer diagnosis. We should take into account the increased risk of atrial fibrillation development and, after this, study the embolic risk and potential indication of oral anticoagulation.

\section{Introduction}

Atrial fibrillation (AF) is the commonest cardiac arrhythmia observed in clinical practice and is associated with significant morbidity and mortality globally. Its prevalence is increasing in part due to an aging population [1], from $\sim 0.5 \%$ in those aged 40 years to $6-12 \%$ in those aged 85 years [2]. Apart from advancing age, independent predictors of AF include hypertension, obesity, diabetes, and smoking $[1,2]$. Moreover, cancer remains the second most common cause of death in the United States. Several risk factors are known to contribute to both $\mathrm{AF}$ and cancer [3]. Thus, mechanistic studies have demonstrated a critical role of proinflammatory states in cancer [4]. A proinflammatory environment has also been linked to $\mathrm{AF}$, as reflected by increases in serum inflammatory biomarkers such as C-reactive protein (CRP), 
interleukin- (IL-) 2, IL-6, and IL-8 [5]. However, evidence from epidemiological studies has been controversial [6-10]. Therefore, this systematic review and meta-analysis was conducted to examine the relationship between cancer and the risk of developing AF.

\section{Methods}

This meta-analysis of observational studies was in accordance with the Strengthening the Reporting of Observational Studies in Epidemiology (STROBE) statement [11].

2.1. Search Strategies. Two researchers (M. Y. and X. F.) systematically and independently searched the relevant studies from the following databases: PubMed (until June 2017) and Web of Science (from 1986 through June 2017). We used the following key words: "cancer," "carcinoma," "tumor," and "atrial fibrillation." The reference lists of the articles, conferences, and editorials were used to identify further relevant studies.

2.2. Inclusion Criteria. All observational studies reported on the epidemiological evidence for an association between solid cancer and AF were included in this meta-analysis. Because the aim of this meta-analysis is to investigate whether cancer patients are at an increased risk of developing AF in general population, study population that involved the patients who underwent surgery and chemotherapy were excluded. The inclusion criteria were articles (1) published in English language, (2) on human subjects, (3) that were case control, prospective or retrospective cohort study, (4) reporting the odds ratios (ORs) or hazard ratios (HRs) and the corresponding 95\% confidence intervals (CIs) or data required for their calculations were provided, (5) assessing the association between AF and cancer, not survival of cancer or complication of AF. Where two articles with overlapping data were included, the articles with higher subjects were included.

2.3. Study Selection. Two researchers (M. Y. and X. F.) independently screened the titles and abstracts of the studies. Potential eligible studies were retrieved using the relevant inclusion criteria mentioned previously. Any disagreements or indeterminations between the two researchers were resolved through discussion or consultation with a third researcher (T. L.).

2.4. Data Extraction and Quality Assessment. Hazard ratio (HRs), odds ratio (ORs), and their 95\% confidence intervals (CIs) for the association between cancer and AF were extracted from individual articles. If both unadjusted and adjusted ORs/HRs were reported, the adjusted ORs/HRs were preferentially used. And we priority to extracted multivariate adjusted ORs/HRs, not age- or gender-adjusted, to evaluate the risk of AF occurrence. The extracted data of each study included first author's last name, year of publication, geographic location of study, study design, total number of subjects, participants' age and sex, types of cancer, criterion for AF confirmation, follow-up duration, maximum adjusted covariates, $\mathrm{HR}$ or OR with $95 \% \mathrm{CI}$, and patients with heart failure, hypertension, and diabetic mellitus.

The Newcastle-Ottawa Scale (NOS) items, with total score of nine stars, were used to evaluate the quality of cohort or case control studies [12]. We defined the cohort or case control studies with NOS score $\geq 7$ stars as high quality and NOS score $<7$ stars as low quality.

2.5. Statistical Analysis. The ORs with 95\% CIs were used as the common risk estimates and then were pooled. The HR value using multivariate Cox proportional hazards model in the original research was directly considered as OR. Percent variability across studies attributable to heterogeneity beyond sampling error was evaluated using the $I^{2}$ statistic, and $I^{2}$ value of $>50 \%$ represented moderate to high heterogeneity. The random effects model was used as it is better to explain heterogeneity between studies over the fixed-effects model. Subgroup analyses and sensitivity analysis were performed to identify the sources of heterogeneity. Subgroup analyses regarding study design (case control studies or cohort studies), study location (Europe and USA), and the methods for AF diagnosis (electrocardiogram and International Classification of Disease code) and time interval between cancer diagnosis and AF were performed. Sensitivity analysis was performed by omitting one study at a time to evaluate the influence of individual studies on the pooled results. The funnel plot was constructed to identify possible publication bias. $P$ values of $<0.05$ (two-tailed) were considered statistically significant. Statistical analyses were performed using the Review Manager (RevMan) software (Nordic Cochrane Center; http://ims.cochrane.org/revman, version 5.3).

\section{Results}

A flow diagram of the data search and study selection is shown in Figure 1. Initially, 6311 records were identified from the PubMed and Web of Science databases. Of these, 2796 were duplicate studies and were excluded. The remaining articles were screened, and 3487 were subsequently excluded because they were review articles, animal studies, or irrelevant to this analysis. The 28 remaining studies were then reviewed in detail, and 23 of the 28 were excluded: study published in Italian, Russian, or Spanish language $(n=5)$ [13-16]; study reported AF leading to cancer $(n=4)$ [17-20]; individual case reports $(n=3)$ [21-23]; letters $[24,25]$ or editorials [26] $(n=3)$; different article from the same center [27, 28], and a more recent series was available [8], patients in this study [29] may be repeated in the study by Conen [10] $(n=3)$; study evaluated the relation of AF and survival in cancer patients $(n=2)$ $[30,31]$; OR was not provided $(n=1)$ [32]; cross-sectional study $(n=1)$ [33]; and study investigated AF as a complication in cancer patients $(n=1)$ [34]. Therefore, a total of 


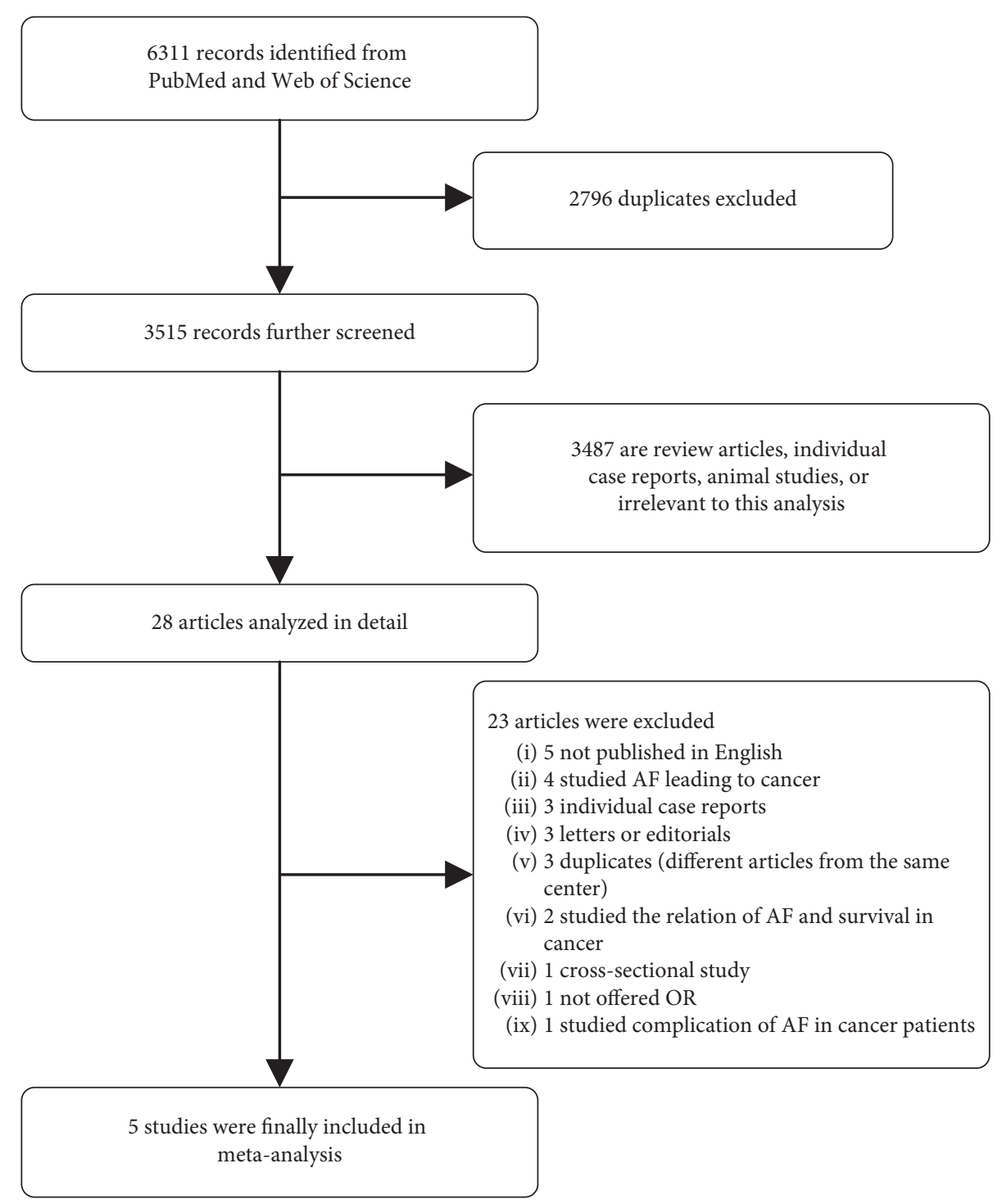

FIgURE 1: Flow diagram of the study selection process. $\mathrm{AF}=$ atrial fibrillation; $\mathrm{OR}=$ odds ratio.

five studies comprising 5,889,234 participants were included in our meta-analysis.

The characteristics of the included studies and their quality scores are shown in Table 1 . Two were case control studies $[7,8]$, and three were cohort studies $[6,9,10]$. Two studies $[6,7]$ investigated only the association between AF and colorectal cancer, whereas three studies [8-10] examined colorectal cancer and also cancer of the breast, lung, and prostate. For the included cohort, the mean age ranged from 53 to 75 years and the proportion of male patients accounted for all patients ranged from $0 \%$ to $60 \%$. Characteristics of the patients are presented in Table 2. NOS analysis showed that all included studies were of high quality.

Apart from one study [6] reporting no significant association between cancer and AF, the remaining four studies [7-10] consistently demonstrated a significant association between cancer and new AF risk. Overall, the summary estimate from the five separate estimates from the cohort or case-control studies united indicated that patients with cancer had an approximately $47 \%$ higher risk of AF compared to noncancer patients (OR 1.47, 95\% CI 1.31 to 1.66 , $p<0.00001$; Figure 2). There was a significant heterogeneity across the studies $\left(I^{2}=67 \%, p=0.02\right)$.

Four of five studies reported OR of incidence of AF in colorectal cancer; the pooled effect sizes of these studies [6-9] (OR 1.54, 95\% CI 1.40 to $1.71, p<0.00001$; heterogeneity: $I^{2}=48 \%, p=0.12$; Figure 3(a)) showed that patients with colorectal cancer at a $54 \%$ higher risk of developing AF than those without colorectal cancer. Two of five studies offered OR of incidence of AF in breast cancer; in the pooled analysis of these studies [8,9] (OR 2.07, 95\% CI 0.96 to 4.45 , $p=0.06$; heterogeneity: $I^{2}=81 \%, p=0.02$; Figure $3(\mathrm{~b})$ ), we observed a prevalence of AF close to two times higher in breast cancer patients compared to those without breast cancer.

Subgroup analyses were subsequently performed to identify potential sources of heterogeneity. The details are 


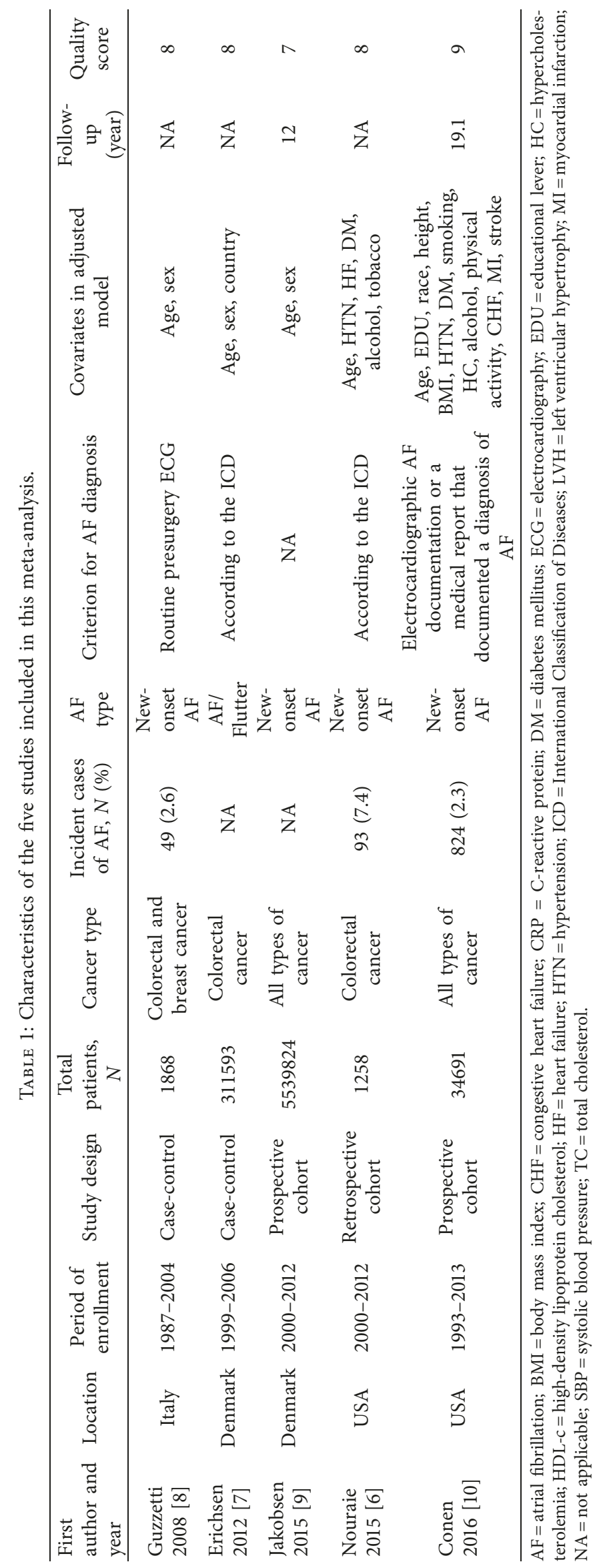


TABle 2: Patient characteristics of the five studies.

\begin{tabular}{|c|c|c|c|c|c|c|c|c|c|c|}
\hline $\begin{array}{l}\text { First } \\
\text { author } \\
\text { and year }\end{array}$ & $\begin{array}{l}\mathrm{AF} \text { in } \\
\mathrm{CA} / \\
\mathrm{NO}- \\
\mathrm{CA} \\
(\%)\end{array}$ & $\begin{array}{c}\text { Mean age, } \\
\text { CA/NO- } \\
\text { CA (year) }\end{array}$ & $\begin{array}{c}\text { Male } \\
\text { sex, } \\
\text { CA/ } \\
\text { NO-CA } \\
(\%)\end{array}$ & $\begin{array}{l}\text { Diabetes, } \\
\text { CA/NO- } \\
\text { CA (\%) }\end{array}$ & $\begin{array}{c}\text { Hypertension, } \\
\text { CA/NO-CA } \\
(\%)\end{array}$ & $\begin{array}{l}\text { Smoking, } \\
\text { CA/NO- } \\
\text { CA (\%) }\end{array}$ & $\begin{array}{c}\text { Cardiovascular } \\
\text { disease, CA/ } \\
\text { NO-CA (\%) }\end{array}$ & $\begin{array}{l}\text { BMI, CA/ } \\
\text { NO-CA }\end{array}$ & $\begin{array}{c}\text { Race, } \\
\mathrm{CA} / \\
\mathrm{NO}- \\
\mathrm{CA}(\%)\end{array}$ & $\begin{array}{c}\text { Education, } \\
\text { high school, } \\
\text { CA/NO-CA } \\
(\%)\end{array}$ \\
\hline $\begin{array}{l}\text { Guzzetti } \\
2008[8]\end{array}$ & $3.6 / 1.6$ & $\begin{array}{c}63.1 \pm 12.6 / \\
63.6 \pm 9.3\end{array}$ & $\begin{array}{c}25.7 / \\
53.0\end{array}$ & NA & NA & NA & NA & NA & NA & NA \\
\hline $\begin{array}{l}\text { Erichsen } \\
2012 \text { [7] }\end{array}$ & NA & $\begin{array}{l}75(70-79) / \\
74(70-79)\end{array}$ & $\begin{array}{l}54.1 / \\
54.1\end{array}$ & $7.1 / 4.5$ & $3.3 / 2.3$ & NA & $37.7 / 21.7$ & NA & NA & NA \\
\hline $\begin{array}{l}\text { Jakobsen } \\
2015 \text { [9] }\end{array}$ & NA & $\begin{array}{c}66.5 \\
(66.5-66.5) / \\
\text { NA }\end{array}$ & $\begin{array}{l}47.4 / \\
\text { NA }\end{array}$ & NA & NA & NA & NA & NA & NA & NA \\
\hline $\begin{array}{l}\text { Nouraie } \\
2015 \text { [6] }\end{array}$ & $\begin{array}{c}10.1 / \\
5.4\end{array}$ & $\begin{array}{l}67(58-78) / \\
56(48-69)\end{array}$ & $47 / 51$ & $33 / 29$ & $59 / 50$ & $27 / 31$ & $14 / 13$ & NA & NA & NA \\
\hline $\begin{array}{l}\text { Conen } \\
2016[10]\end{array}$ & $3.8 / 2.3$ & $\begin{array}{l}55(50-61) / \\
53(49-58)\end{array}$ & $0 / 0$ & $3.2 / 2.6$ & $30.0 / 25.8$ & $53.3 / 47.5$ & NA & $\begin{array}{c}25.1 \\
(22.6-28.9) / \\
24.9 \\
(22.5-28.3) \\
\end{array}$ & $\begin{array}{l}\text { White: } \\
96.5 / \\
94.9\end{array}$ & $43.4 / 44.4$ \\
\hline
\end{tabular}

$\mathrm{CA}=$ cancer group; $\mathrm{NA}=$ not applicable; $\mathrm{NO}-\mathrm{CA}=$ noncancer group.

\begin{tabular}{|c|c|c|c|c|c|c|}
\hline Study or subgroup & Log (odds ratio) & SE & Weight (\%) & $\begin{array}{c}\text { Odds ratio } \\
\text { IV, random, } 95 \% \text { CI }\end{array}$ & $\begin{array}{r}\text { Odc } \\
\mathrm{IV} \text {, rand }\end{array}$ & $\begin{array}{l}\text { ratio } \\
\text { n, } 95 \% \text { CI }\end{array}$ \\
\hline Guzzetti 2008 & 1.203 & 0.3521 & $2.8 \%$ & $3.33(1.67,6.64)$ & & $\longrightarrow$ \\
\hline Nouraie 2015 & 0.3365 & 0.2254 & $6.2 \%$ & $1.40(0.90,2.18)$ & & \\
\hline Conen 2016 & 0.1823 & 0.0879 & $21.9 \%$ & $1.20(1.01,1.43)$ & & - \\
\hline Erichsen 2012 & 0.4511 & 0.0585 & $29.3 \%$ & $1.57(1.40,1.76)$ & & - \\
\hline Jakobsen 2015 & 0.4055 & 0.0068 & $39.8 \%$ & $1.50(1.48,1.52)$ & & $\mathbf{\square}$ \\
\hline Total (95\% CI) & & & $100.0 \%$ & $1.47(1.31,1.66)$ & & \\
\hline \multicolumn{5}{|c|}{$\begin{array}{l}\text { Heterogeneity: tau }{ }^{2}=0.01 ; \mathrm{chi}^{2}=12.27, \mathrm{df}=4(P=0.02) ; I^{2}=67 \% \\
\text { Test for overall effect: } Z=6.35(P<0.00001)\end{array}$} & $\begin{array}{c}0.5 \quad 0.7 \\
\text { Lower risk of } \mathrm{AF}\end{array}$ & $\begin{array}{c}1.5 \quad 2 \\
\text { Higher risk of AF }\end{array}$ \\
\hline
\end{tabular}

FIgURE 2: Forest plot for pooled odds ratio $(\mathrm{OR})$ for the risk of atrial fibrillation (AF) in patients with any cancer. $\mathrm{SE}=$ standard error; IV = inverse variance.

shown in Table 3. Our pooled meta-analysis suggested that cancer is significantly associated with the occurrence of AF in both cohort $[6,9,10]$ and case control $[7,8]$ studies with significant heterogeneity. As shown in Table 3, cancer was associated with an increased risk of AF in both studies originating from Europe [7-9] (OR 1.56, 95\% CI 1.39 to 1.76, $\left.p<0.0001, I^{2}=65 \%\right)$ with significant heterogeneity as well as the United States [6, 10] (OR 1.22, 95\% CI 1.04 to 1.44 , $\left.p=0.01, I^{2}=0 \%\right)$ without heterogeneity.

Subgroup analysis was also performed for the method of AF diagnosis. Meta-analysis of two studies using electrocardiography $[8,10]$ reported a higher risk of $\mathrm{AF}$ in cancer patients (OR $1.89,95 \%$ CI 0.70 to $5.10, p=21, I^{2}=87 \%$ ) with significant heterogeneity. Meta-analysis of two studies that had used the International Classification of Diseases (ICD) $[6,7]$ also demonstrated elevated risk of AF (OR 1.56, 95\% CI $\left.1.39-1.74, p<0.0001, I^{2}=0 \%\right)$, and this was associated with minimal heterogeneity. Therefore, study location [6$10]$ and the method for diagnosing $\mathrm{AF}[6-8,10]$ are both likely the origin of the heterogeneity in our main metaanalysis.

To examine temporal relationship between cancer and $\mathrm{AF}, \mathrm{ORs}$ from two studies were further pooled $[7,10]$ for AF according to the time since cancer was first diagnosed, classified by time interval less than 90 days, 91 to 365 days, or more than 365 days. We found significantly increased risk of $\mathrm{AF}$ in cancer patients diagnosed less than 90 days (OR 7.62, $95 \%$ CI 3.08 to $\left.18.88, p<0.0001, I^{2}=91 \%\right)$. Otherwise, pooled OR of incidence of AF was not significantly increased for longer time-points of 91 to 365 days (OR 1.06, 95\% CI 0.90 to $1.25, p=0.46, I^{2}=0 \%$ ) or beyond 365 days (OR 0.97 , $95 \%$ CI 0.71 to $1.34, p=0.87, I^{2}=84 \%$ ) (Table 3 ). Finally, sensitivity analysis by excluding one study at a time did not significantly alter the pooled OR. The results of the funnel plot for the association between cancer and AF was asymmetry, indicating that publication bias may be present, although the small number of studies made this somewhat difficult to interpret (Figure 4). 


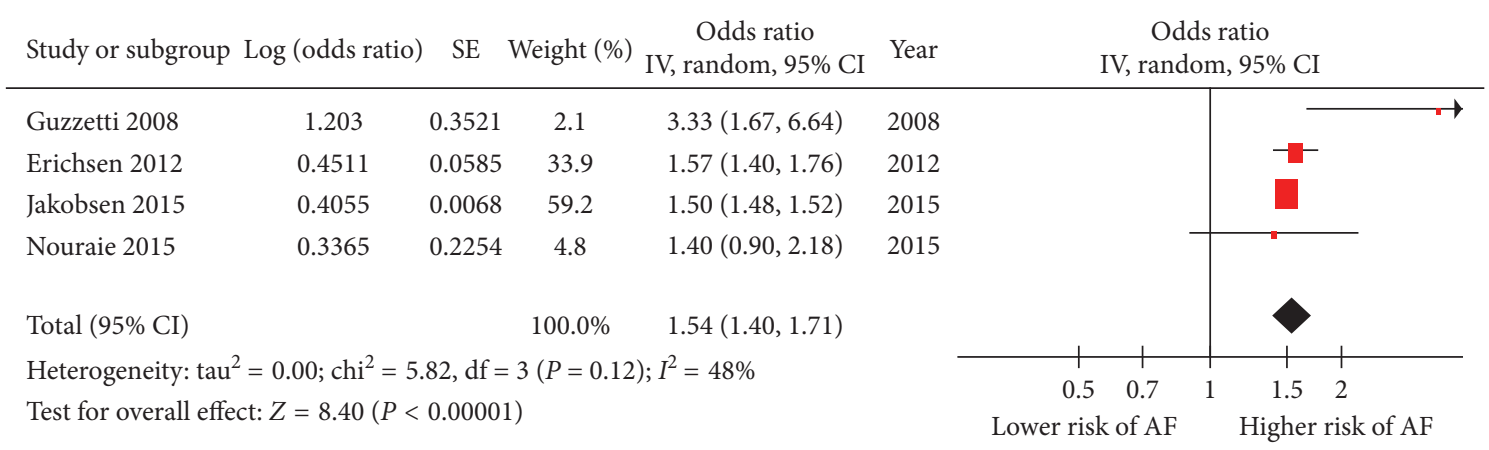

(a)

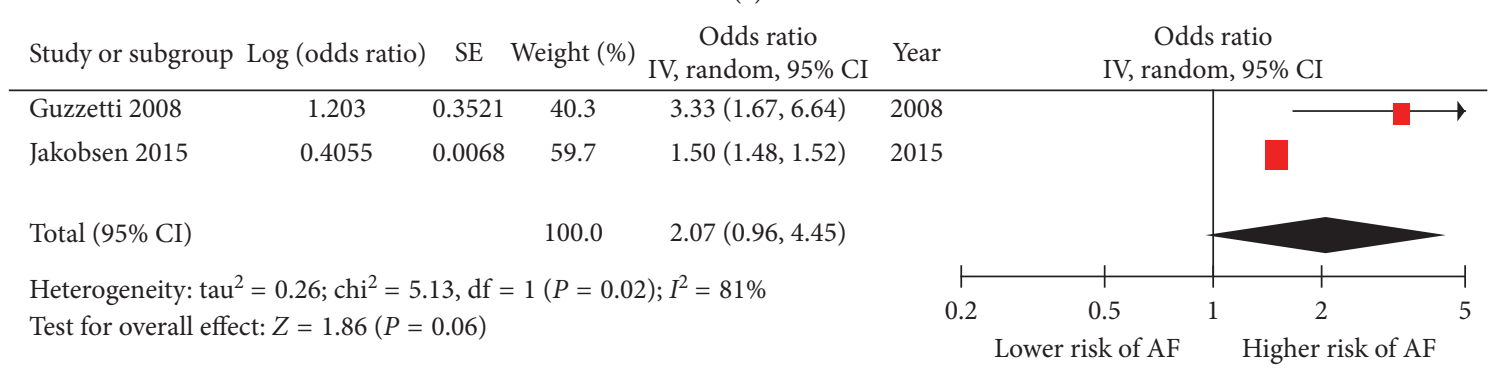

(b)

FIGURe 3: Outcomes for the risk of atrial fibrillation (AF) in patients with different cancer. (a) Forest plot of the odds ratio (OR) for pooled risk of atrial fibrillation (AF) in patients with colorectal cancer; (b) forest plot of the odds ratio (OR) for pooled risk of atrial fibrillation in patients with breast cancer. $\mathrm{SE}=$ standard error; $\mathrm{IV}=$ inverse variance.

TABle 3: Subgroup analysis of the association between cancer and atrial fibrillation.

\begin{tabular}{|c|c|c|c|c|c|c|c|}
\hline & \multirow{2}{*}{ Subgroup } & \multirow{2}{*}{ Number of studies } & \multicolumn{3}{|c|}{ Meta-analysis } & \multicolumn{2}{|c|}{ Heterogeneity } \\
\hline & & & OR & $95 \%$ CI & $p$ value & $I^{2}(\%)$ & $p$ value \\
\hline \multirow{2}{*}{ Study design } & Case control & 2 & 2.11 & $1.03-4.34$ & 0.04 & 77 & 0.04 \\
\hline & Cohort & 3 & 1.38 & $1.15-1.64$ & 0.0004 & 69 & 0.04 \\
\hline \multirow{2}{*}{ Study location } & Europe & 3 & 1.56 & $1.39-1.76$ & $<0.0001$ & 65 & 0.06 \\
\hline & USA & 2 & 1.22 & $1.04-1.44$ & 0.01 & 0 & 0.52 \\
\hline \multirow{2}{*}{ Criterion for AF diagnosis } & ECG & 2 & 1.89 & $0.70-5.10$ & 0.21 & 87 & 0.005 \\
\hline & ICD & 2 & 1.56 & $1.39-1.74$ & $<0.0001$ & 0 & 0.62 \\
\hline \multirow{3}{*}{ Time interval between cancer diagnosis and $\mathrm{AF}$} & $\leq 90$ days & 2 & 7.62 & $3.08-18.88$ & $<0.0001$ & 91 & 0.0009 \\
\hline & $91-365$ days & 2 & 1.06 & $0.90-1.25$ & 0.46 & 0 & 0.48 \\
\hline & $>365$ days & 2 & 0.97 & $0.71-1.34$ & 0.87 & 84 & 0.01 \\
\hline
\end{tabular}

$\mathrm{AF}=$ atrial fibrillation; $\mathrm{CI}=$ confidence interval; $\mathrm{ECG}=$ electrocardiogram; $\mathrm{ICD}=$ International Classification of Disease; $\mathrm{OR}=$ odds ratio.

\section{Discussion}

Our systematic review and meta-analysis of five published observational studies suggests that subjects with newly diagnosed cancer had a significantly increased risk of AF during subsequent follow-up. There was significant heterogeneity observed between the included studies which were likely due to different methods of AF diagnosis. Interestingly, the increased risk of AF was only observed within the first 90 days after cancer diagnosis, and the risk was not significant after 1 year. Our study found substantial statistical heterogeneity in the pooled effect estimates. This is partly explicable by the use of different methods to detect AF in the individual studies. There are some data to support an increased risk of stroke after a cancer diagnosis, and one could hypothesize that this relationship between cancer and
AF could account for part of the elevated risk of stroke if the AF went undetected.

Oncocardiology is a new field of clinical medicine that addresses the close link between cancer and cardiovascular diseases [35, 36]. Recent evidence showed that cancer is closely related to the development of AF. A number of pathophysiological mechanisms, such as inflammation and autonomic dysfunction, have been proposed to explain this link $[5,37]$. Firstly, clinical studies have demonstrated elevations in proinflammatory markers in both AF and cancer. A case-control study showed that patients with atrial arrhythmia compared to those without atrial arrhythmia had higher levels of the inflammatory marker, C-reactive protein (CRP). Indeed, CRP levels were higher in persistent than paroxysmal AF patients [38]. Another large populationbased cohort study reported that CRP was independently 


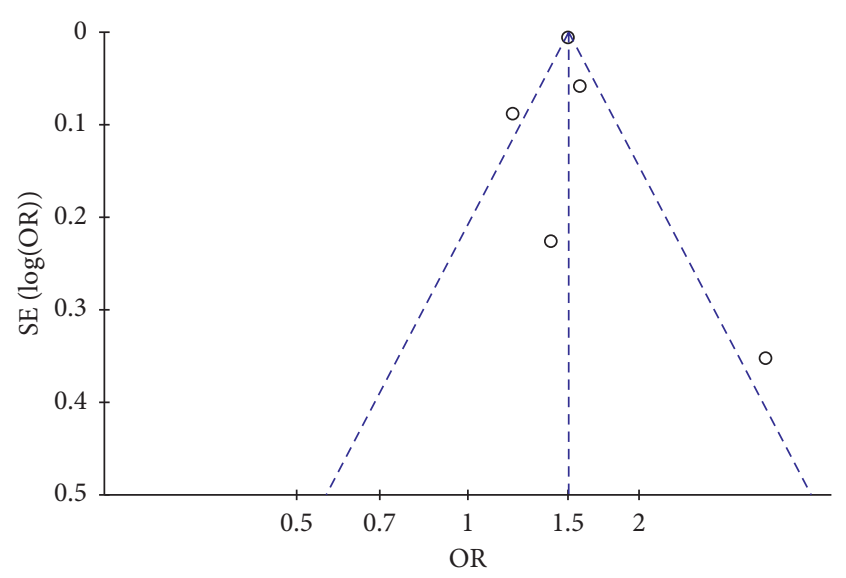

FIGURE 4: Funnel plot for the association between cancer and atrial fibrillation occurrence. $\mathrm{SE}=$ standard error; $\mathrm{OR}=$ odds ratio.

associated with the presence of AF and also predicted patients who were at increased risk of subsequently developing AF [39]. Marcus et al. reported that CRP levels in patients with atrial flutter that were initially elevated fell after successful ablation [40]. These findings are consistent with notion that inflammation plays a key role in structural and electrophysiological modeling that underlies arrhythmic substrates [41]. Similarly, cancer is associated with a proinflammatory state [42]. Thus, CRP levels were significantly elevated in colorectal [43] and breast [44] cancer patients compared to controls. However, in the included studies, data on cancer staging were not available, which could alter the degree of inflammation and the risk of AF.

The second factor is dysfunction of the autonomic nervous system. Altered balance of sympathetic versus parasympathetic activity has been associated with AF [45], and patients with cancer also show some evidence of altered autonomic activity or dysfunction [46]. Pain and emotional or physical stress in cancer may increase sympathetic nervous activity and predispose to atrial fibrillation [47]. Therefore, the patients who diagnosed with cancer underwent mental stress and sympathetic challenge during the first 90 days of receiving such anxiety provoking news which may have predisposed the patients to triggers of AF. Besides, Faber et al. reported that subclinical thyroid diseases seemed to change the structure and function of heart with subsequent alters in morbidity and mortality [48]. And the outcome of another analysis demonstrates that patients with clinical or subclinical hyperthyroidism are at increased risk of AF [49]. A hypothesis concerns that tumors may release thyroid hormones such as thyroid-stimulating hormone (TSH) and triiodothyronine (T3) [8]. Thus, it is possible that cancer enhances the incidence of AF through the abnormal production of thyroid hormones-like peptides [50]. These findings suggest altered autonomic activity in occult cancer, suggesting that undiagnosed cancer may precede AF. Finally, the epidemiological link between AF and cancer may be due to shared risk factors, diagnostic bias, undiagnosed occult cancer leading to AF, and anticoagulation unmasking cancer.
There are several potential limitations of our metaanalysis. Firstly, the data on the epidemiological evidence for the relationship between new-onset cancer and the risk of AF are sparse, and only a few studies have addressed this issue. And given that the total number of studies was small, we included a letter [28] and an abstract of conference [9] to maximize the use of available data. Secondly, some studies only presented the sex- or age-adjusted ORs/HRs, so not all ORs/HRs used in this analysis were extracted from multivariate analysis. This may have introduced a degree of random error in our pooled analysis. Finally, the epidemiological evidence for an association between cancer and risk of AF is mainly investigated in this study, and we will perform further study to uncover the potential relationship between surgery or chemotherapy and development of atrial fibrillation.

In summary, this meta-analysis demonstrates that cancer is associated with atrial fibrillation that was significant within the first three-month period. These findings would prompt us to suggest that AF patients should be screened for occult cancer. Future studies are needed to examine the potential mechanisms linking cancer to $\mathrm{AF}$, and further analyses that can examine the association between $\mathrm{AF}$ and subsequent cancer-related mortality may exclude to possibility of diagnostic bias.

\section{Disclosure}

An earlier version of the manuscript has been presented as conference abstract in Global Cardio-Oncology Summit 2017, available in the following link: http://cardiaconcology.ca/wpcontent/uploads/GCOS2017_029_YXia_Abstract.pdf.

\section{Conflicts of Interest}

The authors declare no conflicts of interest.

\section{Acknowledgments}

I would like to express my gratitude to Dr. Xia and Dr. Liu and all those who helped me during the writing of this article. This work was supported by grants 81570298, 30900618, and 81270245 (to T. L.) from the National Natural Science Foundation of China. G. T. is supported by the Croucher Foundation of Hong Kong.

\section{References}

[1] S. S. Chugh, R. Havmoeller, K. Narayanan et al., "Worldwide epidemiology of atrial fibrillation," Circulation, vol. 129, no. 8, pp. 837-847, 2014.

[2] J. Ball, M. J. Carrington, J. J. V. McMurray, and S. Stewart, "Atrial fibrillation: profile and burden of an evolving epidemic in the 21st century," International Journal of Cardiology, vol. 167, no. 5, pp. 1807-1824, 2013.

[3] D. Farmakis, J. Parissis, and G. Filippatos, "Insights into oncocardiology," Journal of the American College of Cardiology, vol. 63, no. 10, pp. 945-953, 2014.

[4] S. I. Grivennikov, F. R. Greten, and M. Karin, "Immunity, inflammation, and cancer," Cell, vol. 140, no. 6, pp. 883-899, 2010. 
[5] Y. Guo, G. Y. H. Lip, and S. Apostolakis, "Inflammation in atrial fibrillation," Journal of the American College of Cardiology, vol. 60, no. 22, pp. 2263-2270, 2012.

[6] M. Nouraie, V. Kansal, C. Belfonte et al., "Atrial fibrillation and colonic neoplasia in African Americans," PLoS One, vol. 10, no. 8, Article ID e0135609, 2015.

[7] R. Erichsen, C. F. Christiansen, F. Mehnert, N. S. Weiss, J. A. Baron, and H. T. Sørensen, "Colorectal cancer and risk of atrial fibrillation and flutter: a population-based case-control study," Internal and Emergency Medicine, vol. 7, no. 5, pp. 431-438, 2012.

[8] S. Guzzetti, G. Costantino, A. Vernocchi, S. Sada, and C. Fundarò, "First diagnosis of colorectal or breast cancer and prevalence of atrial fibrillation," Internal and Emergency Medicine, vol. 3, no. 3, pp. 227-231, 2008.

[9] C. Jakobsen, N. Carlson, M. Lamberts et al., "Incidence of atrial fibrillation in different types of cancer: a Danish nationwide cohort study," European Heart Journal, vol. 36, p. 164, 2015.

[10] D. Conen, J. A. Wong, R. K. Sandhu et al., "Risk of malignant cancer among women with new-onset atrial fibrillation," JAMA Cardiology, vol. 1, no. 4, pp. 389-396, 2016.

[11] E. von Elm, D. G. Altman, M. Egger, S. J. Pocock, P. C. Gøtzsche, and J. P. Vandenbroucke, "The Strengthening the Reporting of Observational Studies in Epidemiology (STROBE) statement: guidelines for reporting observational studies," The Lancet, vol. 370, no. 9596, pp. 1453-1457, 2007.

[12] A. Stang, "Critical evaluation of the Newcastle-Ottawa scale for the assessment of the quality of nonrandomized studies in meta-analyses," European Journal of Epidemiology, vol. 25, no. 9, pp. 603-605, 2010.

[13] S. Guzzetti, G. Costantino, S. Sada et al., "Atrial fibrillation as a complication of colorectal tumors," Recenti Progressi in Medicina, vol. 94, no. 6, pp. 260-263, 2003.

[14] E. V. Andrushenko, I. I. Polishchuk, T. A. Malanchuk et al., "Atrial fibrillation as the onset of cancer of the left lung," Lik Sprava, vol. 7-8, pp. 128-130, 1995.

[15] C. Alvarez Alvarez, F. Tardaguila Montero, J. A. Carrillo Sande et al., "Atrial fibrillation in an oncological patient with remission," Anales de Medicina Interna, vol. 23, no. 6, pp. 295-296, 2006.

[16] F. Furlanello, R. Miori, E. Piccolo et al., "Paroxysmal atrial arrhythmia and lung neoplasms (clinico-electrocardiographic consideration)," Torace, vol. 17, no. 1-2, pp. 310-325, 1974.

[17] A. D. Müller, A. Sonnenberg, and I. H. Wasserman, "Diseases preceding colon cancer," Digestive Diseases and Sciences, vol. 39, no. 11, pp. 2480-2484, 1994.

[18] S. Fumagalli, A. Barchielli, F. Tarantini et al., "Atrial fibrillation and cancer: evidence for an epidemiological link," Journal of the American College of Cardiology, vol. 59, no. 13, p. E615, 2012.

[19] E. B. Ostenfeld, R. Erichsen, L. Pedersen et al., "Atrial fibrillation as a marker of occult cancer," PLoS One, vol. 9, no. 8, Article ID e102861, 2014.

[20] S. Wassertheil-Smoller, A. P. McGinn, L. Martin et al., "The associations of atrial fibrillation with the risks of incident invasive breast and colorectal cancer," American Journal of Epidemiology, vol. 185, no. 8, pp. 1-13, 2017.

[21] R. Knur and J. Özse, "Atrial fibrillation as the first clinical presentation of an adenoid cystic bronchial carcinoma," Netherlands Heart Journal, vol. 22, no. 10, pp. 472-473, 2014.

[22] A. Beyder and K. W. Klarich, "Large atrial myxoma causing dynamic obstruction of the mitral valve and atrial fibrillation," Mayo Clinic Proceedings, vol. 87, no. 2, p. e9, 2012.
[23] J. E. Cohen, J. Kogan, S. Oren, and M Mazza, "Primary cardiac lymphoma presenting with atrial fibrillation," Israel Medical Association Journal, vol. 13, no. 10, pp. 635-7, 2011.

[24] P. Velagapudi, M. K. Turagam, and A. G. Kocheril, "Atrial fibrillation in cancer patients," Southern Medical Journal, vol. 104, no. 9, pp. 667-668, 2011.

[25] G. P. Koracevic, "Cancer is an insufficiently recognized risk factor for atrial fibrillation," Journal of Emergency Medicine, vol. 42, no. 3, pp. 312-313, 2012.

[26] F. Rahman, D. Ko, and E. J. Benjamin, "Association of atrial fibrillation and cancer," JAMA Cardiology, vol. 1, no. 4, pp. 384-386, 2016.

[27] S. Guzzetti, G. Costantino, and C. Fundarò, "Systemic inflammation, atrial fibrillation, and cancer," Circulation, vol. 106, no. 9, p. e40, 2002.

[28] S. Guzzetti, G. Costantino, S. Sada, and C. Fundarò, "Colorectal cancer and atrial fibrillation: a case-control study," American Journal of Medicine, vol. 112, no. 7, pp. 587-588, 2002.

[29] C. H. Kim, S. G. Al-Kindi, and G. H. Oliveira, "Atrial fibrillation and cancer-validation in the real world," JAMA Cardiology, vol. 2, no. 3, pp. 343-344, 2017.

[30] S. R. Walsh, K. M. Gladwish, N. J. Ward, T. A. Justin, and N. J. Keeling, "Atrial fibrillation and survival in colorectal cancer," World Journal of Surgical Oncology, vol. 2, no. 1, p. 40, 2004.

[31] T.-L. Yang, Y.-F. Hu, Y.-J. Lin et al., “Atrial fibrillation influences survival in patients with hepatocellular carcinoma: experience from a single center in Taiwan," Journal of the Chinese Medical Association, vol. 77, no. 3, pp. 117-121, 2014.

[32] E. Bou, P. Hernandez, L. Cerezo et al., "Heart tumors in Puerto Rico De novo atrial fibrillation as clinical presentation in a subgroup of patients," Puerto Rico Health Sciences Journal, vol. 32, no. 1, pp. 14-17, 2013.

[33] W. T. O’Neal, S. G. Lakoski, W. Qureshi et al., "Relation between cancer and atrial fibrillation (from the REasons for Geographic And Racial Differences in Stroke Study)," American Journal of Cardiology, vol. 115, no. 8, pp. 1090-1094, 2015.

[34] Y.-f. Hu, C.-j. Liu, P. M.-h. Chang et al., "Incident thromboembolism and heart failure associated with new-onset atrial fibrillation in cancer patients," International Journal of Cardiology, vol. 165, no. 2, pp. 355-357, 2013.

[35] W.-L. Cheng, Y.-H. Kao, S.-A. Chen, and Y.-J. Chen, "Pathophysiology of cancer therapy-provoked atrial fibrillation," International Journal of Cardiology, vol. 219, pp. 186194, 2016.

[36] D. Sueta, N. Tabata, T. Akasaka, T. Yamashita, T. Ikemoto, and S. Hokimoto, "The dawn of a new era in onco-cardiology: the Kumamoto Classification," International Journal of Cardiology, vol. 220, pp. 837-841, 2016.

[37] T. Liu, G. Li, L. Li, and P. Korantzopoulos, "Association between C-reactive protein and recurrence of atrial fibrillation after successful electrical cardioversion," Journal of the American College of Cardiology, vol. 49, no. 15, pp. 1642-1648, 2007.

[38] M. K. Chung, D. O. Martin, D. Sprecher et al., "C-reactive protein elevation in patients with atrial arrhythmias," Circulation, vol. 104, no. 24, pp. 2886-2891, 2001.

[39] R. J. Aviles, D. O. Martin, C. Apperson-Hansen et al., "Inflammation as a risk factor for atrial fibrillation," Circulation, vol. 108, no. 24, pp. 3006-3010, 2003. 
[40] G. M. Marcus, L. M. Smith, D. V. Glidden et al., "Markers of inflammation before and after curative ablation of atrial flutter," Heart Rhythm, vol. 5, no. 2, pp. 215-221, 2008.

[41] G. Tse, B. P. Yan, Y. W. Chan et al., "Reactive oxygen species, endoplasmic reticulum stress and mitochondrial dysfunction: the link with cardiac arrhythmogenesis," Frontiers in Physiology, vol. 7, p. 313, 2016

[42] S. J. Wigmore, J. P. Maingay, K. C. H. Fearon, M. G. O'Riordain, and J. A. Ross, "Effect of interleukin-4 on pro-inflammatory cytokine production and the acute phase response in healthy individuals and in patients with cancer or multiple organ failure," Clinical Science, vol. 95, no. 3, pp. 347-354, 1998.

[43] T. P. Erlinger, E. A. Platz, N. Rifai et al., "C-reactive protein and the risk of incident colorectal cancer," JAMA, vol. 291, no. 5, pp. 585-590, 2004.

[44] D. M. O'Hanlon, J. Lynch, M. Cormican et al., “The acute phase response in breast carcinoma," Anticancer Research, vol. 22, no. 2b, pp. 1289-1293, 2002.

[45] Y. Xi and J. Cheng, "Dysfunction of the autonomic nervous system in atrial fibrillation," Journal of Thoracic Disease, vol. 7, no. 2, pp. 193-198, 2015.

[46] R. Martin, J. M. Delgado, J. M. Moltò et al., "Cardiovascular reflexes in patients with malignant disease," Italian Journal of Neurological Sciences, vol. 13, no. 2, pp. 125-129, 1992.

[47] S. Guzzetti, "Systemic inflammation, atrial fibrillation, and cancer," Circulation, vol. 106, no. 9, pp. 40e-40, 2002.

[48] J. Faber and C. Selmer, "Cardiovascular disease and thyroid function," Cardiovascular Issues in Endocrinology, vol. 43, pp. 45-56, 2014.

[49] S. Marrakchi, F. Kanoun, S. Idriss et al., "Arrhythmia and thyroid dysfunction," Herz, vol. 40, no. 2, pp. 101-109, 2015.

[50] L. Mao, W. Huang, P. Zou, X. Dang, and X. Zeng, "The unrecognized role of tumor suppressor genes in atrial fibrillation," Gene, vol. 642, pp. 26-31, 2018. 


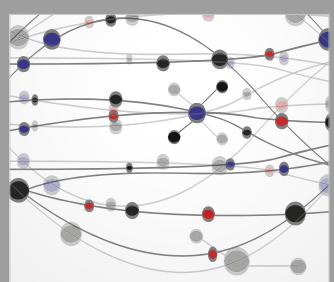

The Scientific World Journal
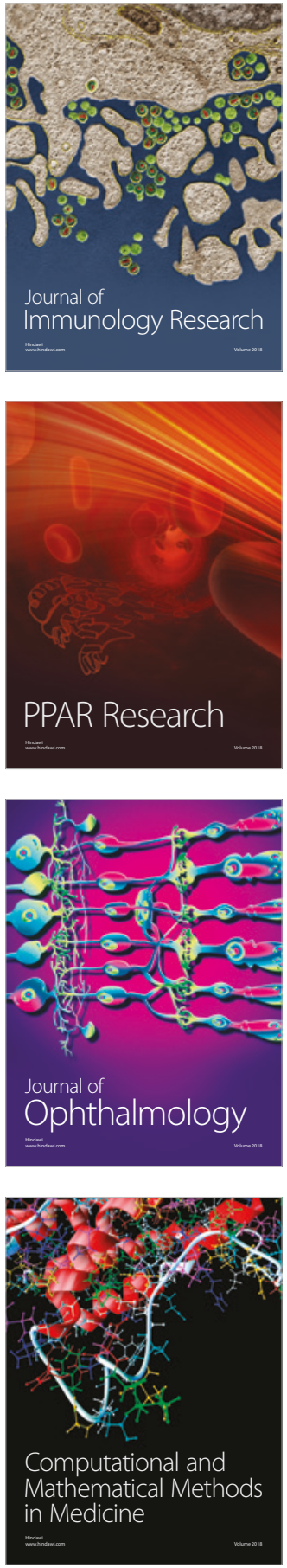

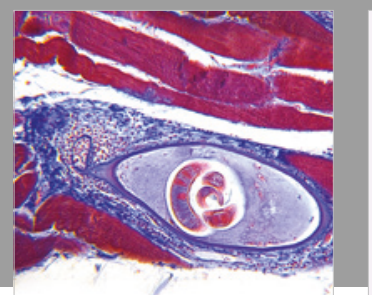

Gastroenterology Research and Practice

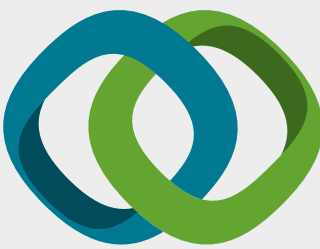

\section{Hindawi}

Submit your manuscripts at

www.hindawi.com
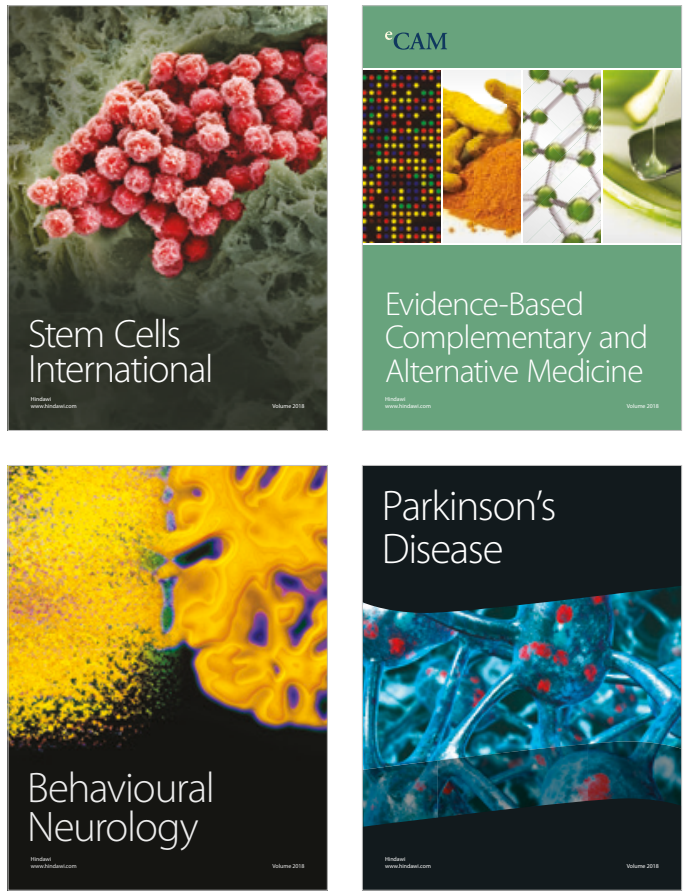

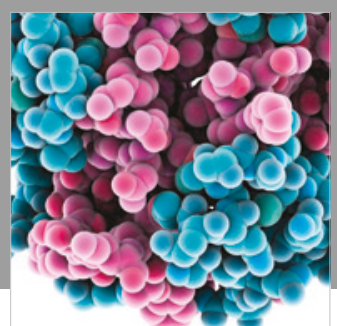

ournal of

Diabetes Research

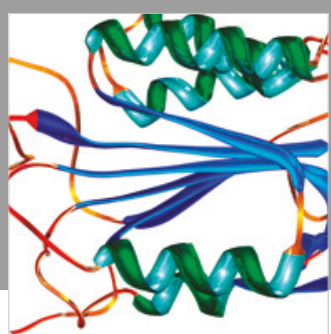

Disease Markers
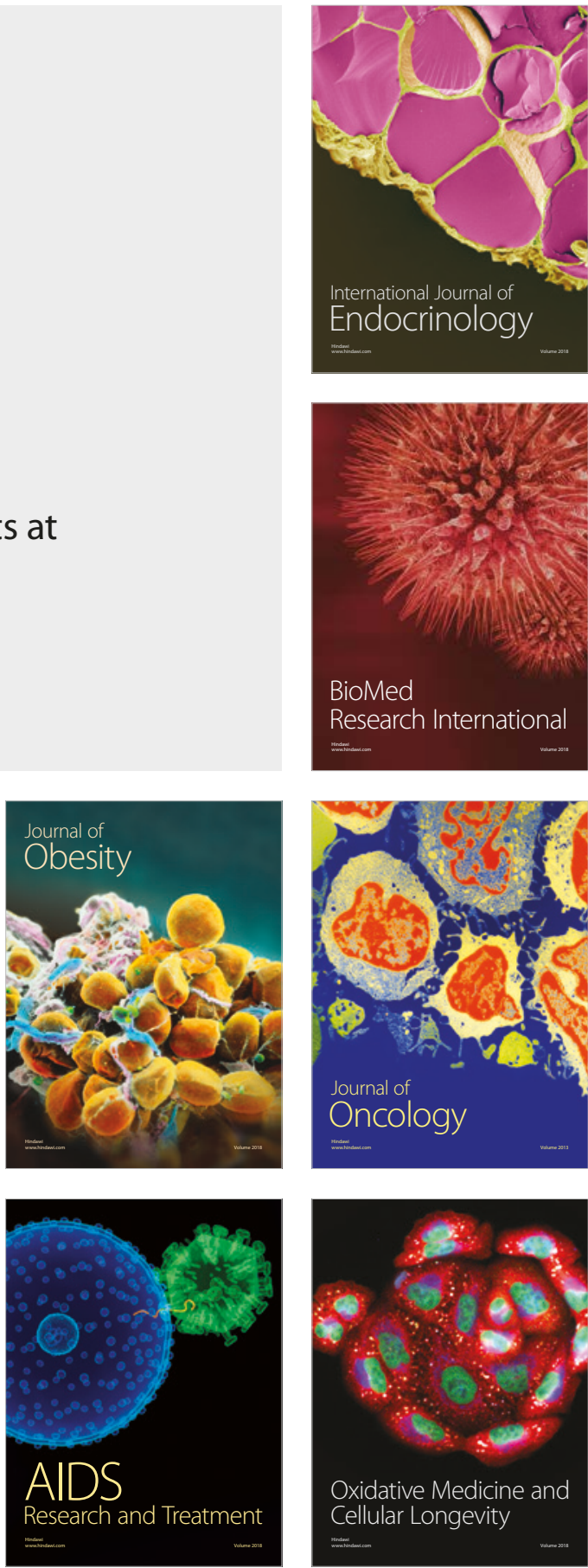